\title{
Reconnection in substorms and solar flares: analogies and differences
}

\author{
J. Birn ${ }^{1}$ and M. Hesse ${ }^{2}$ \\ ${ }^{1}$ Los Alamos National Laboratory, Los Alamos, NM, USA \\ ${ }^{2}$ NASA Goddard Space Flight Center, Greenbelt, MD, USA
}

Received: 8 September 2008 - Revised: 16 February 2009 - Accepted: 16 February 2009 - Published: 4 March 2009

\begin{abstract}
Magnetic reconnection is the crucial process in the release of magnetic energy associated with magnetospheric substorms and with solar flares. On the basis of threedimensional resistive MHD simulations we investigate similarities and differences between the two scenarios. We address in particular mechanisms that lead to the onset of reconnection and energy release, transport, and conversion mechanisms. Analogous processes might exist in the motion of field line footpoints on the sun and in magnetic flux addition to the magnetotail. In both cases such processes might lead to a loss of neighboring equilibrium, characterized by the formation of a very thin embedded current sheet, which acts as trigger for reconnection. We find that Joule (or ohmic) dissipation plays only a minor role in the overall energy transfer associated with reconnection. The dominant transfer of released magnetic energy occurs to electromagnetic energy (Poynting) flux and to thermal energy transport as enthalpy flux. The former dominates in low-beta, specifically initially force-free current sheets expected for the solar corona, while the latter dominates in high-beta current sheets, such as the magnetotail. In both cases the outflow from the reconnection site becomes bursty, i.e. spatially and temporally localized, yet carrying most of the outflow energy. Hence an analogy might exist between bursty bulk flows (BBFs) in the magnetotail and pulses of Poynting flux in solar flares. Further similarities might exist in the role of collapsing magnetic flux tubes, as a consequence of reconnection, in the heating and acceleration of charged particles.
\end{abstract}

Keywords. Magnetospheric physics (Magnetospheric configuration and dynamics; Storms and substorms) - Space plasma physics (Magnetic reconnection)

Correspondence to: J. Birn

(jbirn@lanl.gov)

\section{Introduction}

Analogies between magnetospheric substorms and solar eruptions, specifically coronal mass ejections (CMEs) and solar flares, have been invoked for a long time (e.g., Kuperus, 1976). A central process in either case is the release of previously stored magnetic energy and its conversion into particle energy in the form of heating, bulk plasma kinetic energy, and accelerated particles with suprathermal energies. Also the ejection of a plasmoid or flux rope is a central part of substorms as well as CMEs. In this paper we focus on additional similarities that may exist in the mechanisms for initiating reconnection and the details of the energy release and conversion processes.

We start out in Sect. 2 with a brief description of the basic approach and the numerical procedure, followed by simulation results on the early phase preceding the onset of reconnection (Sect. 3), and an overview of the subsequent dynamic evolution, initiated by finite resistivity (Sect. 4). We then investigate the energy transfer after the onset of reconnection, based on three-dimensional MHD simulations (Sect. 5), complemented by more localized, two-dimensional particlein-cell simulations of reconnection (Sect. 6). The mechanisms that govern dissipation and transfer are addressed in more detail in Sect. 7.

\section{Initial states and numerical procedure}

Our initial states are derived from the explicit threedimensional equilibrium models given by Birn et al. (2003). These models are valid for configurations that vary most rapidly in one spatial direction (here, $x$, corresponding to the magnetospheric $z$ coordinate). This is a reasonable approximation for the magnetotail and, presumably, also for the stretched field configuration below a departing CME. However, the field closer to Earth and to the solar surface can

Published by Copernicus Publications on behalf of the European Geosciences Union. 


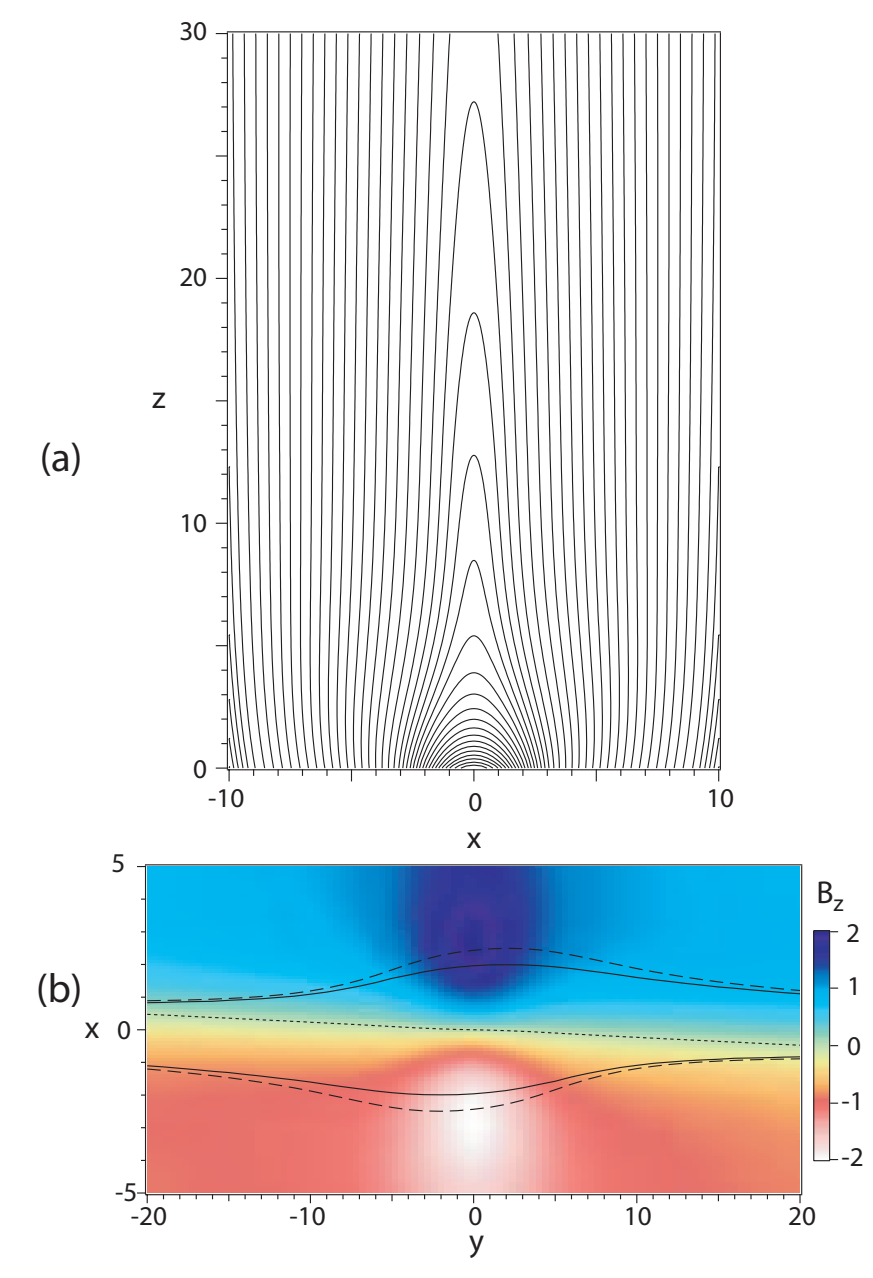

Fig. 1. (a) Initial magnetic field in the $x, z$ plane and (b) the colorcoded magnitude of the normal magnetic field $B_{z}$ at the bottom boundary $z=0$ for a force-free initial state after relaxation. The dotted line indicates $B_{z}=0$. The dashed and solid lines show the boundaries of field lines that close within the box before and after the foot point motion, respectively. Note that the full integration box size is given by $|x| \leq 10,|y| \leq 40$, and $0 \leq z \leq 60$.

be expected to be less stretched. Therefore we added a 3-D dipole magnetic field with a location of the dipole below the lower boundary of the simulation box. Since this superposition leads to fields that are no longer in exact force balance, we used a relaxation method (Hesse and Birn, 1993) to obtain force balanced equilibria before starting the simulation of the temporal evolution.

The stretched field configurations contain a current sheet separating magnetic fields of roughly opposite directions. Two types of current sheet configurations are considered. One is initially force-free, that is, the current density vector is aligned with the magnetic field. In this type of configuration, the pressure of the magnetic field that reverses across the current sheet, $B_{z}$, is balanced by the magnetic pressure of the component in the perpendicular direction, $B_{y}$, in the di- rection of the peak current in the center of the sheet, such that the magnitude of $B_{y}$ in the center equals the magnitude of the reconnecting field component outside the current sheet. This field is commonly denoted the "guide field". Note, that the magnitude of the guide field decreases away from the center in force-free configurations, approaching zero in our chosen configurations. Strictly force-free fields require uniform pressure, such that the pressure gradients vanish. In the other case, the guide field is assumed to be small or zero and the outside magnetic pressure is balanced by the plasma pressure inside the current sheet. This configuration is typical for the Earth's magnetic tail. A small uniform background plasma pressure of $1 \%$ of the magnetic pressure is added in all cases. The force-free and the non-force-free initial states are therefore also distinguished by low and high plasma $\beta$ in the current sheet ( $\beta=$ ratio of plasma over magnetic pressure), whereas the background has always small $\beta=0.01$. This value appears reasonable above solar active regions (e.g., Gary, 2001) as well as for the magnetotail lobes.

Figure 1a shows the initial magnetic field in the $x, z$ plane and Fig. $1 \mathrm{~b}$ the color-coded magnitude of the normal magnetic field component $B_{z}$ at the boundary $z=0$ for the forcefree initial state after relaxation. The dotted line in Fig. 1b indicates the neutral line $B_{z}=0$. The dashed lines show the boundaries of field lines that close within the box. As discussed in the following section, a converging footpoint motion is applied at $z=0$ in an early, quasistatic phase, to foster current density intensification and thereby the onset of reconnection. The displaced boundary is indicated by the solid lines. The converging motion leads to an increase in the boundary field strength $B_{z}$ of about $70 \%$.

The field evolution is integrated using a one-fluid MHD code (e.g., Birn et al., 1996, 2006). A nonlinear grid is used to increase the resolution in the regions of interest, such that about $1 / 2$ of the grid points lie within the current sheet. As for the equilibrium, gravity is neglected. We further neglect radiation and heat conduction, assuming an adiabatic law with a ratio of specific heats $\gamma=5 / 3$ but including Ohmic heating. This assumption seems reasonable, as we focus on the energy release powering the impulsive phase of substorms or flares. We note that in our one-fluid code, mass density and pressure represent the sum of electron and ion (here simply proton) density and pressure.

Here and in the following we use dimensionless units, based on a characteristic magnetic field strength $B_{c}$, a scale length $L_{c}$, and a density $n_{c}$ (or equivalently a velocity $v_{c}$ ). For illustration and quantitative comparison with solar observations, we will use

$B_{c}=0.01 \mathrm{~T}=100 \mathrm{G}$
$L_{c}=10000 \mathrm{~km}$
$n_{c}=2 \times 10^{15} \mathrm{~m}^{-3}$

(corona)

These values lead to a velocity unit (Alfvén speed) $v_{c}=5000 \mathrm{~km} / \mathrm{s}$, time unit $t_{c}=L_{c} / v_{c}=2 \mathrm{~s}$, and energy unit $k T_{c}=B_{c}^{2} / \mu_{0} n_{c}=4 \times 10^{-14} \mathrm{~J}=250 \mathrm{keV}$. 
For magnetotail application we choose

$B_{c}=40 \mathrm{nT}$

$L_{c}=10000 \mathrm{~km} \approx 1.5 R_{E}$

$v_{c}=1000 \mathrm{~km} / \mathrm{s} \quad$ (magnetotail)

leading to $t_{c}=10 \mathrm{~s}$ and $k T_{c}=1.6 \times 10^{-13} \mathrm{~J}=10 \mathrm{keV}$. We use (mostly) standard international (SI) units with common notations with $k$ being the Boltzman constant. Our coordinate system is chosen such that $x$ is the coordinate perpendicular to the current sheet (horizontal axis in Fig. 1a), $y$ is in the direction of the main current (perpendicular to the plane of Fig. 1a), and $z$ is the vertical direction, different from usual magnetospheric coordinates.

The boundary conditions consist of solid, ideally conducting walls at each of the boundaries $z=0, y= \pm y_{\max }= \pm 40$, and $x= \pm x_{\max }= \pm 10$, where all velocity components are set to zero (except for the initial phase, discussed below). Von Neumann boundary conditions $(\partial / \partial n=0)$ are imposed on density, pressure, and the tangential magnetic field components, except at the top boundary $z=60$, where an open outflow condition is assumed, such that $B_{x}$ and $B_{y}$ are convected with the plasma flow, while the normal magnetic field is held fixed. Line symmetry conditions are imposed at $x=0, y=0$. For most runs, the number of grid cells in $x, y$, and $z$, are 64, 80, and 132, respectively, for the full box. By varying the grid size and the resolution we confirmed that the basic results reported here are robust.

\section{Early evolution leading to initiation of reconnection}

The stretched arcade configuration, illustrated in Fig. 1, does not automatically lead to the onset of reconnection. In fact, we now know that the onset of reconnection in collisionless plasmas requires thin current sheets with a thickness of about one ion inertial length or less (e.g., Drake and Shay, 2007; Hesse, 2007) and a reduction of the normal magnetic field component ( $B_{x}$ in Fig. 1a) to almost zero (e.g., Pritchett, 2007). In contrast, the typical current sheet thickness in the magnetotail is of the order of several tens of thousands $\mathrm{km}$ and the current sheet in the stretched field below a departing CME probably has similar thickness, far above the ion inertia length. Additional thinning therefore is required before reconnection can start at a significant rate.

One mechanism to accomplish that has been suggested by Birn and Schindler (2002), based on analytic theory and numerical modeling of stretched configurations as those of Fig. 1a. They demonstrated that a relatively small but nonuniform compression of such current sheet configuration, satisfying ideal MHD constraints, can lead to a critical state, where a neighboring equilibrium ceases to exist. The critical configuration is characterized by the formation of a very thin embedded current sheet extending into a cusp type magnetic field structure. At the cusp the thickness of the embedded current sheet and the magnitude of the normal magnetic field go to zero, hence satisfying the onset conditions for fast collisionless reconnection.

An important factor in this scenario is the conservation of entropy on deformed field lines, which can be associated with the conservation of an integral

$S=\int p^{1 / \gamma} d s / B$

where $s$ denotes the arc length along a field line and the integration is from one footpoint of the field line to the other. In two-dimensional force-free configurations, a similar conservation law can be written for the displacement of footpoints of field lines in the invariant $(y)$ direction.

$Y=\int B_{y} d s / B$

A converging motion in the transverse $(x)$ direction does not affect the integral (4), such that an evolution of force-free fields under the conservation of the displacement $Y$ for each field line should lead to a similar critical state, loss of equilibrium, and onset of reconnection.

Our simulations therefore include a slow phase of current intensification prior to the initiation of reconnection. In this phase we apply a slow converging motion at the bottom boundary $z=0$ in the $x$ direction towards the field reversal near $x=0$. This motion, with a maximum amplitude at $y=0$, is gradually turned on and off, as described in detail by Birn et al. (2009). During this phase, the resistivity is set to zero, allowing current to build under the action of the slow driving.

Figure 2 shows results of this evolution, demonstrating the intensification of the current density for the initially forcefree case. The intensified current forms a very thin layer embedded within a wider region of more moderate current density. We should note that the force-free initial configuration does not remain exactly force-free, as a consequence of the motion. However, the plasma $\beta$ remains below $\sim 0.025$.

\section{Brief overview of the dynamic evolution}

In this section we provide a brief overview of the dynamic evolution. More details can be found in Birn et al. (2009). The eruptive phase is initiated by imposing a localized finite resistivity, centered near the peak of the current intensification, but kept fixed in time. The maximum value $\eta_{0}=0.005$ of the resistivity corresponds to a Lundquist number (magnetic Reynolds number) of 200, based on the units of the initial state. This resistivity, in combination with the current intensification from the previous phase leads to the onset of reconnection.

Snapshots of the dynamic evolution of the magnetic field are illustrated by Fig. 3, which shows perspective views of magnetic field lines. Figure 3 a corresponds to a force-free initial state, while Fig. $3 \mathrm{~b}$ represents a high- $\beta$ case with a small but finite shear field. Reconnection leads to an ejection of a part of the closed field lines in the form of a flux 

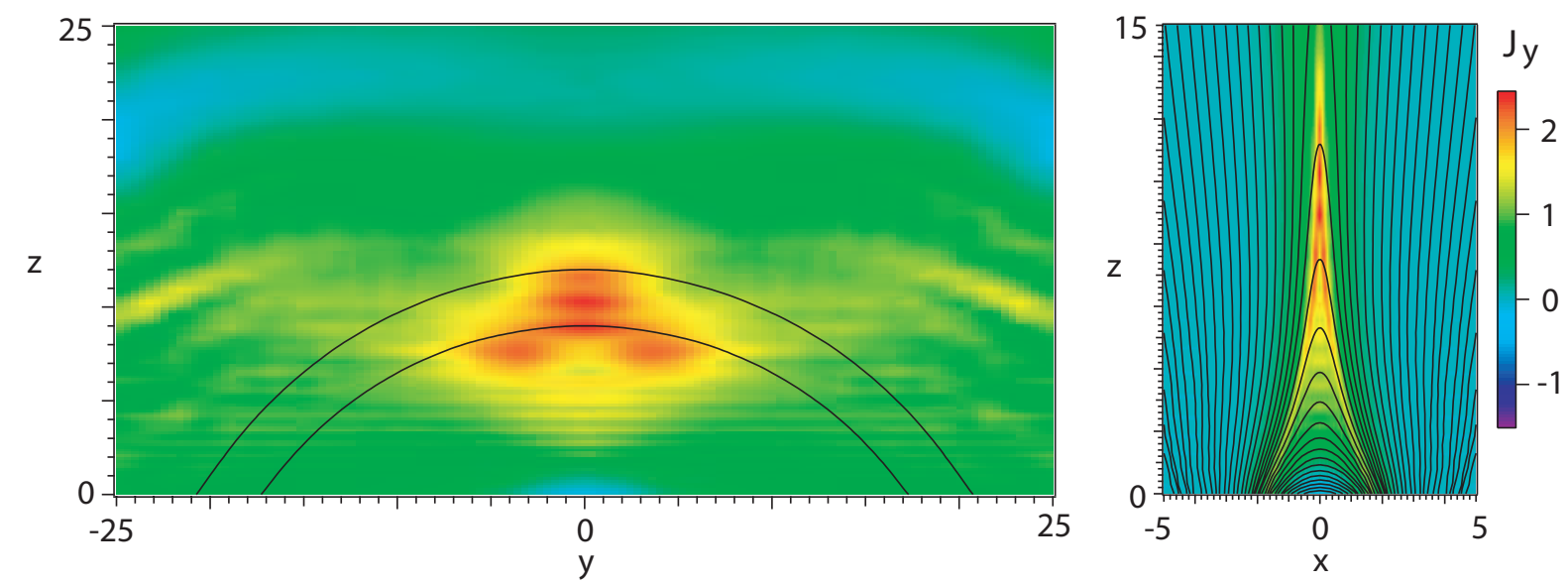

Fig. 2. (a) Current density $J_{y}$ and magnetic field lines in the $y, z$ plane and (b) current density $J_{y}$ and magnetic field in the $x, z$ plane for the force-free case after completion of the footpoint motion.

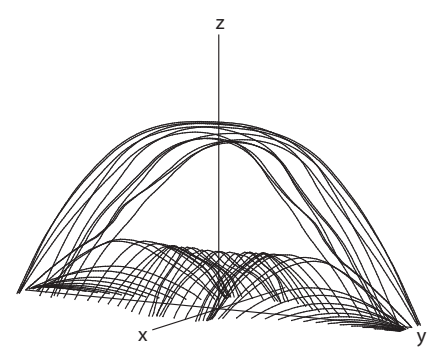

(a)

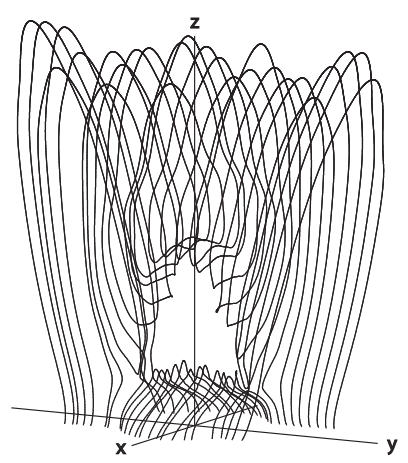

(b)
Fig. 3. Perspective view of magnetic field lines during plasmoid ejection, for (a) a force-free initial state and (b) a high- $\beta$ case with a very small shear field.

rope or plasmoid, while the field below the reconnection site collapses. The high- $\beta$ case, Fig. 3b, shows a tightly wound flux rope, whereas the departing flux rope in Fig. $3 \mathrm{a}$ is only slightly twisted.

\section{Energy conversion and transfer}

In this section we investigate the properties of energy conversion and transport associated with the reconnection process, focussing particularly on the conversion to energy downflow. In the one-fluid, MHD model there are three types of energy flux, the Poynting vector $\mathbf{S}$, describing electromagnetic energy flux

$\mathbf{S}=\boldsymbol{E} \times \boldsymbol{B} / \mu_{0}$

the enthalpy flux, describing the convective transport of thermal energy,

$\mathbf{H}=(u+p) \boldsymbol{v}$ where $u=p /(\gamma-1)$ is the internal (thermal) energy density and the contribution $p \boldsymbol{v}$ describes the effects of compressional work, and the bulk kinetic energy flux

$\mathbf{K}=\frac{1}{2} \rho v^{2} \boldsymbol{v}$

Figure 4 illustrates the characteristic energy transfer for both low and high $\beta$ cases at the time of fastest reconnection, showing energy fluxes in the $x, z$ plane: (from left to right) the Poynting vector components $S_{x}$ and $S_{z}$, the enthalpy flux component $H_{z}$, and the kinetic energy flux component $K_{z}$, respectively. The figure clearly shows that incoming Pointing flux ( $S_{x}$, Fig. 4 , first panels from the left) is mostly converted to up and down going Poynting flux $\left(S_{z}\right.$, Fig. 4 , second panels) and up and down going enthalpy flux $\left(H_{z}\right.$, Fig. 4 , third panels). The kinetic energy flux (Fig. 4, right panels) mostly goes into the upward direction. In the region downward from the reconnection site, the Poynting flux dominates in the low- $\beta$, initially force-free, case (Fig. 4a), whereas the enthalpy flux dominates in the high- $\beta$ case (Fig. 4b). The dominance of the downgoing Poynting flux in the low- $\beta$ case results from the strong guide field, which does not participate in reconnection and simply dominates the redirected electromagnetic energy flux. The strong guide field also makes the plasma less compressible and thereby reduces the effects of compressional heating (see Sect. 7). However, this effect diminishes as reconnection proceeds and the strength of the guide field in the incoming plasma decreases.

The relative proportions of outgoing Poynting flux and enthalpy flux depend on the plasma $\beta$ of the initial state. This is demonstrated by Fig. 5 which shows the temporal evolution of the energy release and transfer, (a) for the force-free initial state, and (b) for a shear-free initial state. The fluxes are integrated over the surfaces of a box $-2 \leq x \leq 2,4 \leq z \leq 15$, $|y| \leq y_{\max }$ surrounding the reconnection site (indicated by red rectangles in Fig. 4). They enter from the sides $|x|=2$, and 

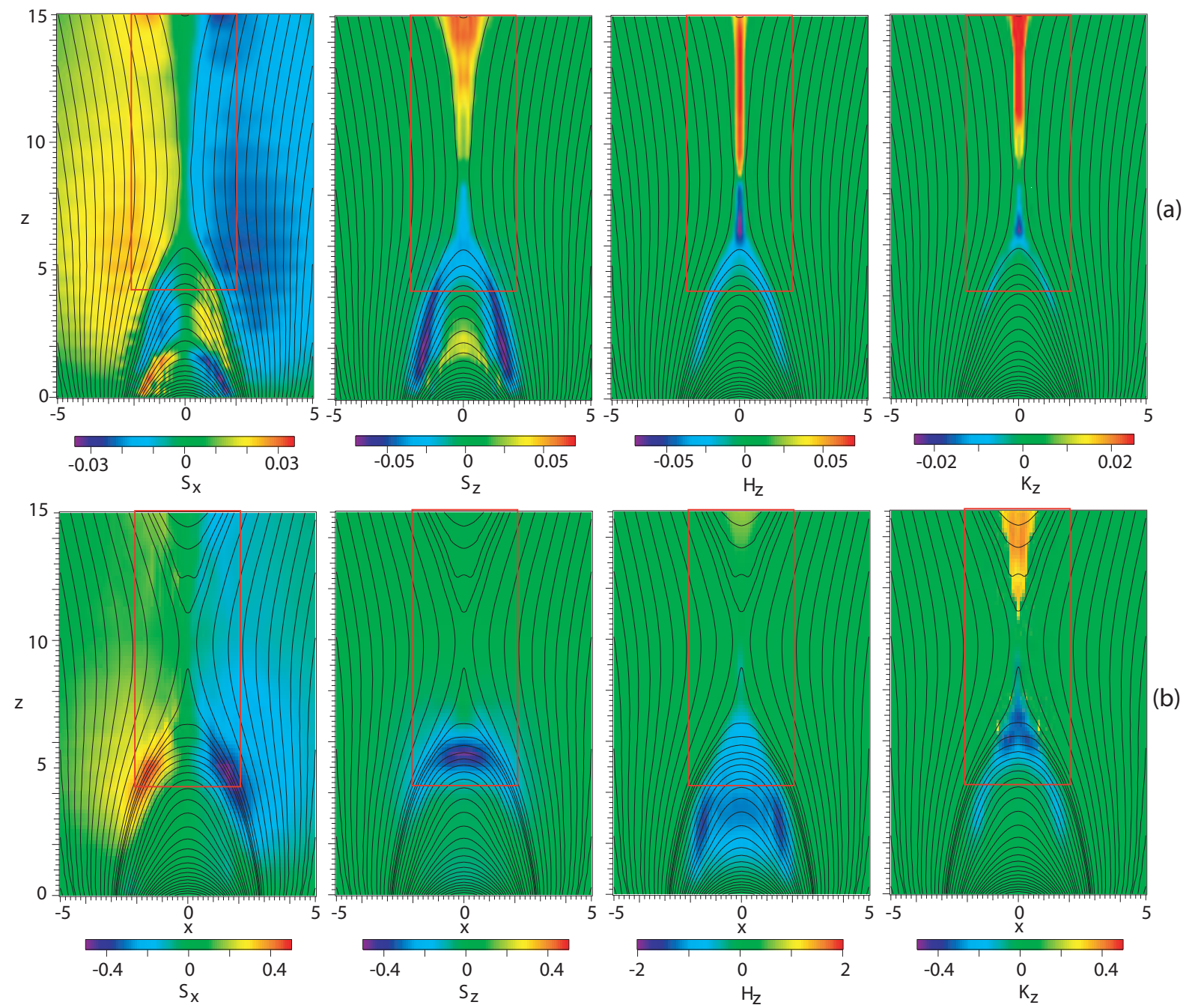

Fig. 4. Energy transfer for (a) a low- $\beta$, initially force-free, case and (b) a high- $\beta$ low shear case, showing energy fluxes in the $x, z$ plane: (from left to right) the Poynting vector components, $S_{x}$ and $S_{z}$, the enthalpy flux component $H_{z}$, and the kinetic energy flux component $K_{z}$. The red rectangles indicate the boundaries of a box used to integrate incoming and outgoing energy fluxes, to be shown in Fig. 5 .

go out through the top, $z=15$, and bottom, $z=4$. The solid lines show the Poynting flux $S_{x}$ into the reconnection site, the blue-colored lines the Poynting flux $S_{z}$ up- and downward from the reconnection site, the purple-colored lines the up- and downward enthalpy fluxes $H_{z}$, and the red-colored line the upward bulk kinetic energy flux. The incoming enthalpy and kinetic energy fluxes, as well as the downgoing kinetic energy flux are very small and therefore not included. We further note that the in and out going fluxes do not perfectly balance. This is primarily due to a decrease of magnetic energy within the box, which results from the fact that the incoming Poynting flux carries smaller guide field, and hence less magnetic energy than the outgoing Poynting flux.

Figure 5 demonstrates that the process of energy release and conversion to downgoing energy mainly consists of a redirection of Poynting flux, originating from the release of magnetic energy, and the conversion to enthalpy flux, resulting from compression and, to a minor extent, from Ohmic dissipation. In the (initially) force-free case the downward Poynting flux dominates over the enthalpy flux, whereas the opposite is true for the shear-free case with the high- $\beta$ current sheet. Note that even though the plasma $\beta$ in the force-free case was initially only 0.01 , a substantial enthalpy flux of about 20\% of the Poynting flux is obtained in the downward fluxes.

The energy fluxes are highly variable in time with multiple peaks of $\sim 10$ Alfvén times duration, corresponding to $\sim 20 \mathrm{~s}$ in our solar application and $\sim 100 \mathrm{~s}$ in the magnetotail application. This time scale is quite commensurate with that of the impulsive phase of solar flares and with flow bursts and the early substorm expansive phase in the magnetotail (although observations in both cases often also show fluctuations on 


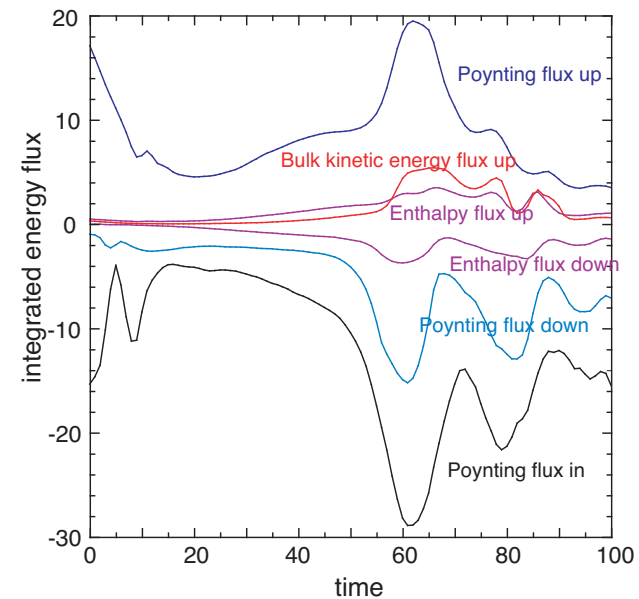

(a)

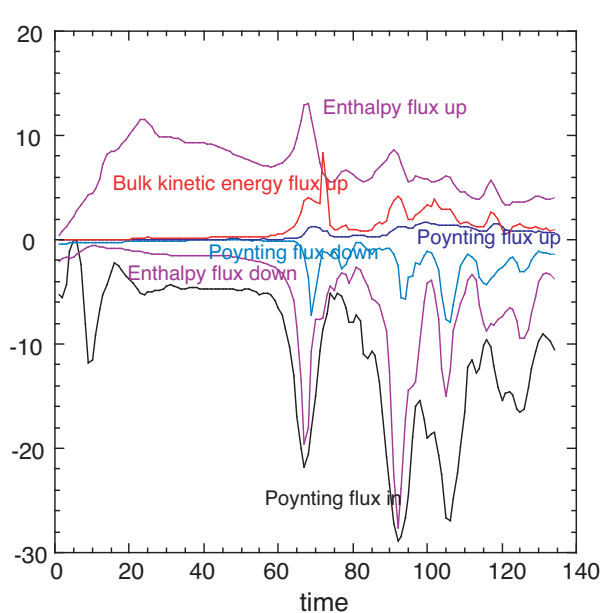

(b)

Fig. 5. Temporal evolution of energy fluxes into and out of the reconnection site, integrated over the surfaces of a box shown but the red rectangles in Fig. 4; (a) force-free initial state, (b) shear-free initial state. The solid black lines show the Poynting flux $S_{x}$ into the reconnection site, the blue-colored lines the Poynting flux $S_{z}$ up- and downward from the reconnection site, the green-colored lines the upand downward enthalpy flux $H_{z}$, and the red-colored line the upward bulk kinetic energy flux.
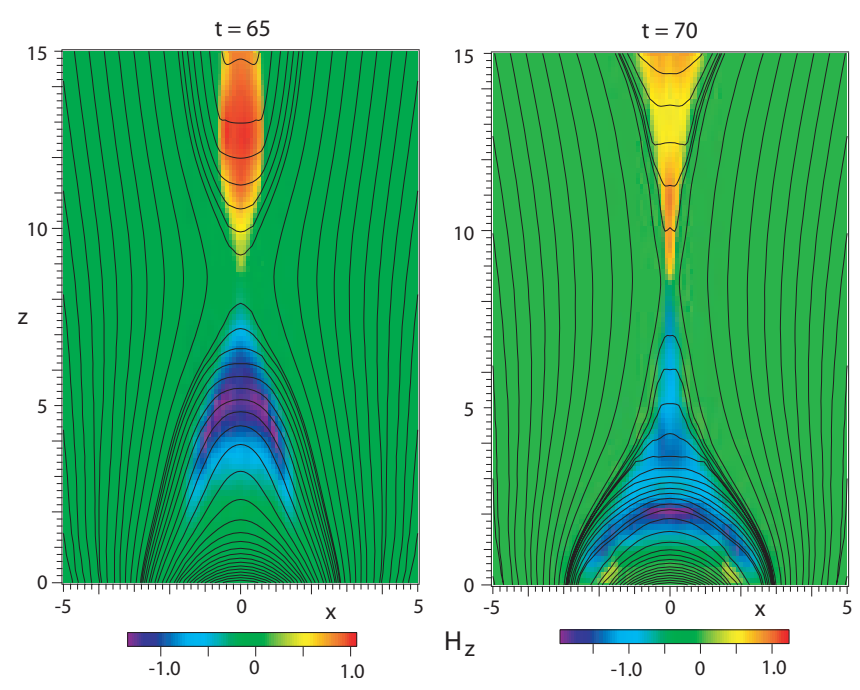

Fig. 6. Enthalpy flux $H_{z}$ in the $x . z$ plane at two successive times for a high- $\beta$ case, demonstrating the association between the enthalpy flux transport, related to a bursty bulk flow, and the collapse of the magnetic field.

shorter time scales not contained in the MHD model). Apparently the time scale is set by the characteristic dimensions of the configuration in the downflow region. We find that the decrease of the magnitude of the fluxes after the first peak coincides with an increase in reflected fluxes from the bottom boundary. The time scale therefore appears to be controlled by the length scale and the wave propagation speed, say, Alfvén speed, both of which are quite similar for flare and substorm scenarios.
Applying our chosen dimensional units (1) and (2) to the fluxes in Fig. 5, we obtain for the solar case a unit of $5 \times 10^{22} \mathrm{~W}=5 \times 10^{29} \mathrm{erg} / \mathrm{s}$, leading to peak downward energy fluxes of the order of $10^{24} \mathrm{~W}=10^{31} \mathrm{erg} / \mathrm{s}$ in bursts of $\sim 20$ $40 \mathrm{~s}$ duration. These numbers are also quite commensurate with those for the impulsive phase of large flares. For the magnetotail application we obtain peak energy fluxes of $\sim 2 \times 10^{12} \mathrm{~W}$ in bursts of $\sim 100$ s duration, again commensurate with the estimated energy release during substorm expansion (e.g., Baker et al., 1997).

The energy flux pulses are related to velocity pulses and the collapse of the magnetic field below the reconnection site. This is clearly demonstrated by Fig. 6 which shows the enthalpy flux $H_{z}$ in the $x . z$ plane for a high- $\beta$ case at two successive times. We do not show the corresponding pictures for the low- $\beta$ case with a strong guide field, because the velocity pulse is more Alfvénic, traveling more closely along the magnetic field and thus out of a plane $y=$ const.

\section{Energy conversion in a PIC simulation}

As stated earlier, reconnection in both the magnetotail and the solar corona presumably involves kinetic processes and dissipation mechanisms that differ considerably from those in resistive MHD (e.g., Hesse, 2007). This might also have considerable effects on the energy partitioning. We have therefore investigated the energy transfer also in particle simulations, albeit in a more localized 2.5-dimensional simulation. The simulations used a mass ratio $m_{i} / m_{e}=25$ and a temperature ratio $T_{i} / T_{e}=5$ with a background pressure of $20 \%$ of the total magnetic pressure $(\beta=0.2)$. As in the so-called Newton challenge problem (Birn et al., 2005), 
reconnection was initiated by localized compression, maximum at $z=0$, applied over a finite time. In contrast to the Newton challenge, a finite guide field was included here, using a force-free initial state. In this case the magnitude of the guide field is equal to the reconnecting field in the center of the current sheet but decreases to zero on the outside.

Figure 7 shows the results from a simulation that started from a one-dimensional current sheet with an initial halfthickness of $2 \lambda_{i}$, where $\lambda_{i}=c / \omega_{p i}$ is the ion inertial length. Details of these simulations will be presented elsewhere. Here we note that the pressure in the PIC simulation does not necessarily remain isotropic and includes both ion and electron contributions. To enable a comparison with the onefluid MHD results, we used definitions of $u$ and $p$ as $1 / 2$ and $1 / 3$ of the trace of the pressure tensor, respectively, i.e.

$u=\frac{1}{2} \sum_{j=1}^{3} P_{j j} \quad p=\frac{1}{3} \sum_{j=1}^{3} P_{j j}$

where $P_{j k}$ represents the sum of proton and electron pressure tensors in the center of mass frame.

Similar to our MHD simulation analysis we have integrated in and outgoing energy fluxes in the PIC simulation at the time of fastest reconnection, using a box around the reconnection site indicated by the red rectangles in Fig. 7. This led to the following net results

$$
\begin{aligned}
& S_{\text {in }}=4.38 \quad S_{\text {out }}=3.87 \\
& H_{i \text { in }}=1.15 \quad H_{i \text { out }}=2.46 \\
& H_{e \text { in }}=0.28 \quad H_{e \text { out }}=2.00 \\
& K_{\text {in }}=0.37 \quad K_{\text {out }}=0.51
\end{aligned}
$$

For comparison we have also treated the localized Newton challenge problem for the force-free case using a MHD simulation with the same parameters. The net results of energy transfer at the time of fastest reconnection are listed below

$$
\begin{aligned}
S_{\text {in }}=0.92 & S_{\text {out }}=0.36 \\
H_{\text {in }}=0.40 & H_{\text {out }}=0.72 \\
K_{\text {in }}=0.01 & K_{\text {out }}=0.24
\end{aligned}
$$

The MHD numbers are typically smaller than those for the PIC simulation. This is largely due to the fact that the reconnection rate was smaller, due to a modest resistivity with a maximum value $\eta_{\max }=0.005$. Qualitatively, however, MHD and PIC simulation results are similar, showing a dominant role of conversion to enthalpy flux, rather than bulk kinetic energy flux. There is a reduction in Poynting flux but also a significant component that just becomes redirected. This is even more pronounced in the PIC simulation.

Significant differences are demonstrated by Fig. 7: The outflow in the PIC simulation is typically one-sided; the electrons gain a substantial increase of enthalpy flux (this cannot be addressed with MHD); and the outgoing ion and electron flows exit to different sides along the magnetic field. The outgoing electron flow may be considered as a field-aligned
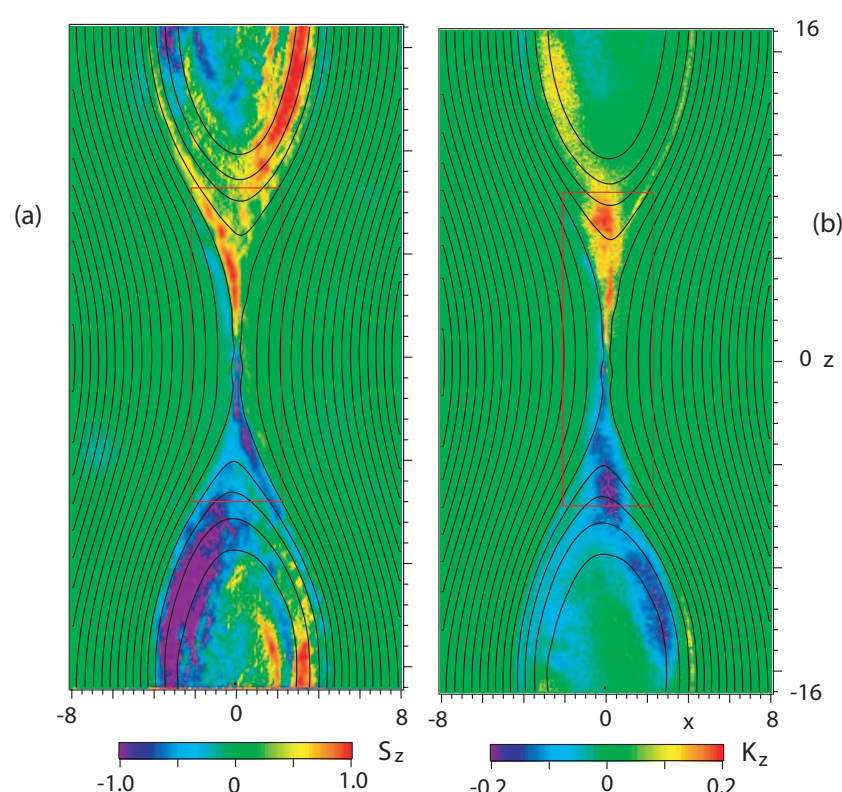

(b)
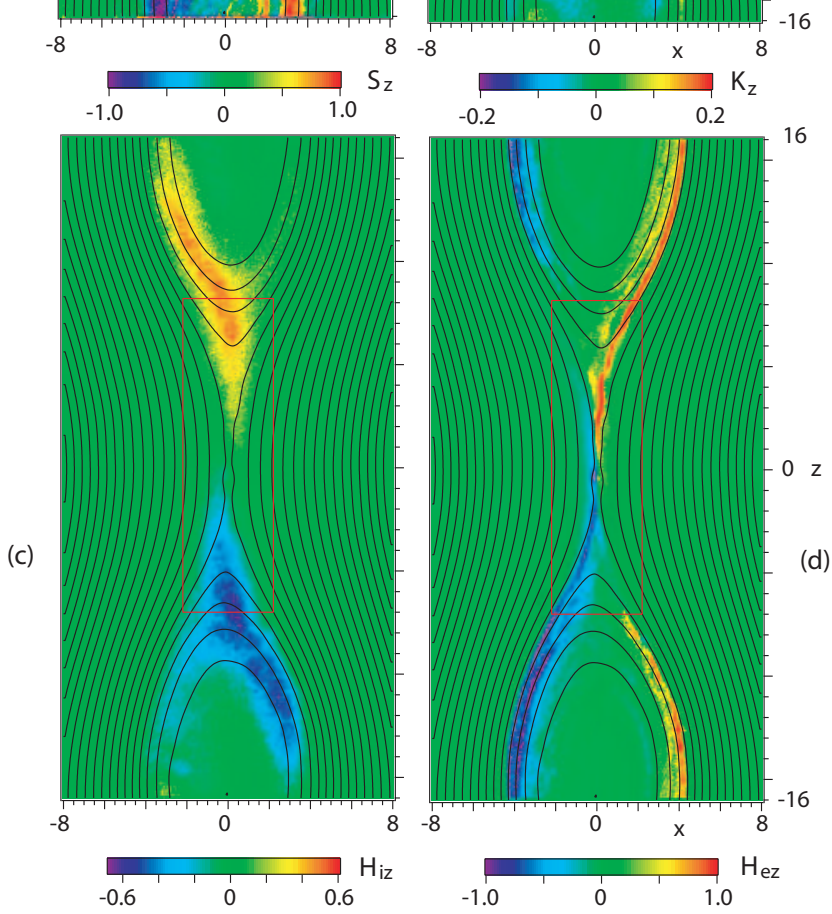

Fig. 7. Energy outflow in a particle-in-cell simulations of magnetic reconnection, showing (a) Poynting flux $S_{z}$, (b) ion enthalpy flux $H_{i z}$, and (c) electron enthalpy flux $H_{e z}$, and (d) bulk kinetic energy flux $K_{z}$. The red rectangles indicate the boundaries of a box used to integrate incoming and outgoing energy fluxes discussed in the text.

beam. However, in contrast to the standard flare acceleration beam model, this beam has high temperature (or energy density) but only modest bulk speed, so that the energy is contained in the enthalpy flux, rather than the bulk kinetic energy flux. Here we should also note that the division of energies between ions and electrons can be expected to be affected by the (artificially high) mass ratio $m_{e} / m_{i}$ in the PIC simulations and by the initial temperature ratio, which is not well known in the solar case. 


\section{Dissipation and heating}

In the previous sections we have presented results on the energy transfer without discussing the mechanisms that govern the transfer and the dissipation. To do this it is useful to discuss energy equations for the individual parts in a form that is applicable to both MHD and particle simulations, as given, for instance, by Birn and Hesse (2005). These equations are generalized here to include heat flux and anisotropic pressure. Electromagnetic energy release and transport is governed by Poynting's theorem (neglecting electric field energy)

$$
\begin{aligned}
\frac{\partial}{\partial t} \frac{B^{2}}{2 \mu_{0}} & =-\nabla \cdot\left(\frac{\boldsymbol{E} \times \boldsymbol{B}}{\mu_{0}}\right)-\boldsymbol{j} \cdot \boldsymbol{E} \\
& =-\nabla \cdot\left(\frac{\boldsymbol{E} \times \boldsymbol{B}}{\mu_{0}}\right)-\boldsymbol{j} \cdot \boldsymbol{E}^{\prime}-\boldsymbol{v} \cdot(\boldsymbol{j} \times \boldsymbol{B})
\end{aligned}
$$

where $\boldsymbol{E}^{\prime}$ is the electric field in the plasma rest frame defined by

$$
\boldsymbol{E}^{\prime}=\boldsymbol{E}+\boldsymbol{v} \times \boldsymbol{B}
$$

In addition there is internal (thermal) energy transport, governed by

$$
\frac{\partial u}{\partial t}=-\nabla \cdot[u \boldsymbol{v}+\mathbf{P} \cdot \boldsymbol{v}+\boldsymbol{Q}]+\boldsymbol{v} \cdot(\nabla \cdot \mathbf{P})+\boldsymbol{j} \cdot \boldsymbol{E}^{\prime}
$$

where $u$ is defined by Eq. (8), $\boldsymbol{Q}$ represents heat flux and $\mathbf{P}$ is the (full) pressure tensor. The first two terms in the square bracket represent the generalized form of enthalpy flux (Eq. 6). The third form of energy is bulk kinetic energy, governed by

$$
\frac{\partial}{\partial t} \frac{\rho}{2} v^{2}=-\nabla \cdot\left(\frac{\rho}{2} v^{2} \boldsymbol{v}\right)+\boldsymbol{v} \cdot(\boldsymbol{j} \times \boldsymbol{B}-\nabla \cdot \mathbf{P})
$$

In these equations we have summed electron and ion contributions, neglecting terms of order $m_{e} / m_{i}$, to ease the comparison between PIC and MHD simulations.

The (resistive) MHD approximations consist of assuming isotropic pressure, neglecting heat flux $\boldsymbol{Q}$, and using Ohm's law, $\boldsymbol{E}^{\prime}=\eta \boldsymbol{j}$. In contrast, in the collisionless model that underlies PIC simulations $\boldsymbol{E}^{\prime}$ is given by (e.g., Vasyliūnas, 1975)

$$
\boldsymbol{E}^{\prime}=\frac{1}{n e} \boldsymbol{j} \times \boldsymbol{B}-\frac{1}{n e} \nabla \cdot \mathbf{P}_{e}+\frac{m_{e}}{n e^{2}}\left[\frac{\partial \boldsymbol{j}}{\partial t}+\nabla \cdot(\boldsymbol{j} \boldsymbol{v}+\boldsymbol{v} \boldsymbol{j})\right]
$$

The first term on the right-hand side of Eq. (15), the Hall term, does not contribute to dissipation, $\boldsymbol{j} \cdot \boldsymbol{E}^{\prime}$. Therefore the remaining two terms, both of which result from electron inertia, are responsible for collisionless dissipation (e.g., Hesse, 2007).

There are three different terms that govern the transfer of energy from one form to another. The first one is Joule dissipation, $\boldsymbol{j} \cdot \boldsymbol{E}^{\prime}$, equivalent to Ohmic heating $\eta j^{2}$ in the resistive MHD model which is by definition positive. In a collisionless model, however, this term need not be positive everywhere. Joule dissipation (if positive) leads to a direct transfer from magnetic to thermal energy. The second term, $\boldsymbol{v} \cdot(\boldsymbol{j} \times \boldsymbol{B})$, represents acceleration (or deceleration) by Lorentz forces as a mechanism of transfer between magnetic and bulk kinetic energy, while the third term, $\boldsymbol{v} \cdot(\nabla \cdot \mathbf{P})$ provides the transfer between bulk kinetic energy and thermal energy, representing work done by pressure forces. For isotropic pressure $p$ this term becomes reduced to $\boldsymbol{v} \cdot \nabla p$. We note that in approximate force balance, $\boldsymbol{j} \times \boldsymbol{B} \approx \nabla \cdot \mathbf{P}$, the combination of these two terms also provide a mechanism of transfer between magnetic and thermal energy. This is in fact how compressional heating may transfer magnetic to thermal energy also in collisionless plasmas. While this is in principle a reversible process it may contribute to irreversible heating when combined with the unidirectional transport from inflow to outflow in reconnection. We note that the heat flux $Q$ does not contribute to energy transfer. It acts as a means to redistribute thermal energy, particularly along field lines, where heat conductivity is much larger than across the field.

To identify the relative importance of the transfer terms we have integrated these terms over the box indicated by the red rectangle in Fig. 7, obtaining

$$
\begin{aligned}
D_{J} & =\int \boldsymbol{j} \cdot \boldsymbol{E}^{\prime} d F=1.16 \\
D_{L} & =\int \boldsymbol{v} \cdot(\boldsymbol{j} \times \boldsymbol{B}) d F=2.09 \\
D_{P} & =\int \boldsymbol{v} \cdot(\nabla \cdot \mathbf{P}) d F=1.87
\end{aligned}
$$

where $d F=d x d z$, and we have used Eq. (12) for $\boldsymbol{E}^{\prime}$, $\boldsymbol{j}=n e\left(\boldsymbol{v}_{i}-v_{e}\right)=\nabla \times \boldsymbol{B} / \mu_{0}$, and the full pressure tensor $\mathbf{P}$. This demonstrates that there is an approximate balance between the effects of the Lorentz forces (Eq. 17) and pressure gradient forces (Eq. 18), such that compressional heating dominates over direct Joule heating (Eq. 16) and thus is primarily responsible for the increase in enthalpy flux.

The units for the integrated transfer terms are the same as those for the fluxes integrated over the boundaries of or reference box, enabling a direct comparison. In an approximate steady state one would expect

$$
\begin{aligned}
\Delta S & =S_{\text {out }}-S_{\text {in }}=-0.51 \approx-D_{J}-D_{L}=-3.25 \\
\Delta H & =H_{\text {out }}-H_{\text {in }}=3.02 \approx D_{J}+D_{P}=3.03 \\
\Delta K & =K_{\text {out }}-K_{\text {in }}=0.14 \approx D_{L}-D_{P}=0.22
\end{aligned}
$$

when the divergence of heat flux is neglected in Eq. (20). To check the effects of heat flux we have calculated the third order moments of the ion and electron distribution functions and integrated the combined heat flux in the same fashion as the other energy fluxes to obtain $\Delta Q=0.44$ as a net outflow of heat flux. This value reduces the (incidental) almost exact agreement between the two estimates of the thermal fluxes in Eq. (20). The major discrepancy, however, shown in Eq. (19), is the fact that the reduction in Poynting flux is 
much smaller than expected from the transfer terms. This, as well as the other differences, is due to the fact that the system is not in a steady state. Particularly the magnetic energy within the reference box decreases in time, primarily from a decrease in the strength of the guide field, due to the fact that the strength of the guide field in the outflow region is larger than the strength of the guide field in the inflow region, which continuously decreases in time. Overall, however, the transfer terms confirm the results obtained from the energy fluxes: the negligible role of conversion to bulk kinetic energy and the dominance of conversion to enthalpy flux, primarily from compression, and the redirection of Poynting flux.

\section{Summary and conclusions}

Using ideal and resistive MHD simulations, we have investigated similarities and differences between magnetic reconnection scenarios in the magnetotail and the solar corona, related to magnetospheric substorms and solar flares. The initial configurations consisted of stretched closed field line models typical for the magnetotail and, in the solar case, representing the expected structure below a departing CME (e.g., Forbes, 1996). Since it is not clear whether the current sheet in the stretched field configuration below the CME is force-free, and for comparison with the magnetotail, we have investigated both force-free and non-force-free configurations. The latter require a finite plasma pressure within the current sheet, whereas the force-free case is characterized by a strong shear field (guide field) in the direction of the peak current. In both cases we have assumed a low plasma $\beta=0.01$ in the outside region, typical for both the corona and the magnetotail lobes.

The highly stretched fields of our initial states enclose elongated current sheets of finite thickness. In the magnetotail the typical thickness of the plasma/current sheet is of the order of $10000 \mathrm{~km}$ and the length several tens or even hundreds of thousands of $\mathrm{km}$. Similar dimensions can be expected in the stretched field below the departing CME. From particle simulations of collisionless reconnection (e.g., Drake and Shay, 2007; Hesse, 2007; Pritchett, 2007) and direct observations in the magnetotail (e.g., Baumjohann and Nakamura, 2007), however, we know that much more severe current sheet thinning to about one ion inertia length is required for the onset of fast collisionless reconnection. This dimension is a few hundreds of $\mathrm{km}$ in the tail and even much less in the solar corona. We have therefore argued that additional drastic thinning is required to enable the onset of fast reconnection.

A possible mechanism exists in the deformation of the field configuration from relatively modest changes in boundary conditions under ideal MHD constraints (Birn and Schindler, 2002). Such changes might lead to a situation where neighboring equilibria satisfying the constraints cease to exist (loss of equilibrium). This concept has been invoked as a possible mechanism for CMEs for quite a while (e.g., Birn et al., 1978; Forbes, 1990; Low, 1996). For the magnetotail, it has been demonstrated that changes resulting from the addition of magnetic flux to the tail might lead to such a loss of equilibrium and that the critical state is characterized by the formation of a very thin current sheet (infinitely thin in the ideal MHD limit), embedded in the wider plasma/current sheet (Birn and Schindler, 2002). This thin current sheet obviously can also enable the onset of fast reconnection.

In the solar case, a similar thin current sheet formation can result from a converging footpoint motion in the already stretched field. Similar effects might also be expected to result from vortical flows in the wake of a departing CME. We have therefore included in our simulations an initial phase during which such localized footpoint motion was imposed, equivalent to the compression of the magnetotail field from flux addition to the near-Earth tail lobes. This indeed caused the formation of thin embedded current sheets with significantly enhanced current density and reduced thickness.

Reconnection was then initiated by imposing finite resistivity, using an ad hoc model to break the frozen-in condition and enable dissipation and reconnection. In more realistic, collisionless, models of reconnection, the dissipation results primarily from electron inertia which causes nongyrotropy of the electron pressure tensor (e.g., Vasyliūnas, 1975; Hesse et al., 1999, 2001). However, the fact that the dissipation is highly localized in our simulations and contributes only negligibly to the overall energy conversion suggests that the MHD results may be relevant more generally even if the actual dissipation is not resistive. A comparison of the MHD results with the dissipation in a (more localized) particle simulation of reconnection supports this view.

The onset of reconnection from the finite resistivity leads to the ejection of a part of the closed field lines in the form of more or less tightly wound flux ropes and a collapse of the field below the reconnection site. This scenario represents substorm features as well as a three-dimensional extension of the standard CSHKP model of two-ribbon flares (Carmichael, 1964; Sturrock, 1966; Hirayama, 1974; Kopp and Pneuman, 1976).

Using these simulations we have then investigated the energy release and conversion, contrasting low and high- $\beta$ current sheets, or equivalently, force-free and shear-free initial configurations. For both, low and high- $\beta$, current sheets we found that the dominant energy transfer downward consists of a redirection of Poynting flux (originating from the release of magnetic energy in the corona or the magnetotail lobes) and a conversion to enthalpy flux, that is, convected quasi-thermal energy, whereas bulk kinetic energy flux is significant only in the outward direction. The increase in enthalpy flux results primarily from compressional heating, rather than Joule dissipation.

In the low- $\beta$ case, the downgoing Poynting flux is the dominant contribution, whereas the enthalpy flux dominates in the high- $\beta$ case. The dominance of the downgoing 


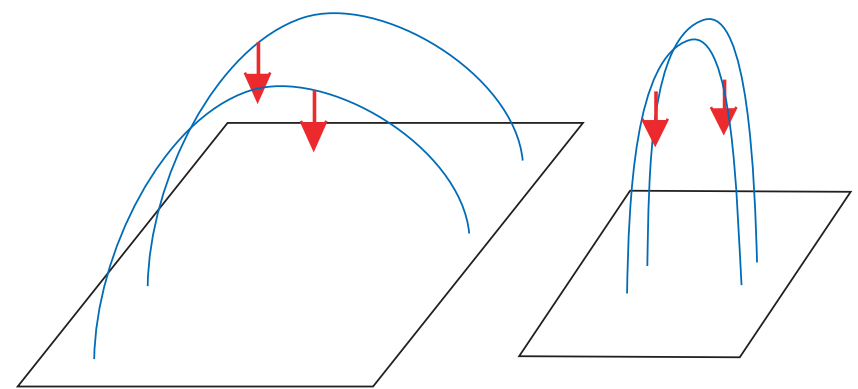

(a)

(b)

Fig. 8. Sketch of the effect of velocity pulses for (a) large guide field and (b) small or zero guide field. Blue lines are magnetic field lines and red arrows indicate a velocity pulse In case (a) the pulse is primarily Alfvénic, perpendicular to the magnetic field, while in case (b) the pulse, away from the apex of a loop, is more strongly field-aligned, associated with a compressional wave.

Poynting flux in the low-beta case is consistent with an alternative to the thick target electron beam model for solar flare energy transport, suggested recently by Fletcher and Hudson (2008). The dominance of Poynting and enthalpy fluxes over the bulk kinetic energy flux, and the dominant role of compression, rather than Joule dissipation in the increase in enthalpy flux, was also confirmed in a, more localized, PIC simulation, using a mass ratio $m_{i} / m_{e}=25$. However, the PIC simulations also indicated asymmetries in the outflow and differences between electron and ion outflow not included in the MHD simulations.

Even in low- $\beta$ cases we found that compressibility and related heating play an important role. This can be understood from the fact that, in a force-free current sheet, the guide field maximizes in the center of the current sheet and decreases outward. Consequently, as reconnection proceeds, the guide field that is transported toward the reconnection site decreases in time and the plasma pressure at the reconnection site must increase to maintain approximate pressure balance with the exterior magnetic pressure. Further pressure increase results from the compression of downward collapsing reconnected flux tubes.

The energy transport down from the reconnection site occurs in a bursty fashion, associated with spatially and temporally localized plasma flows. The flows arise from an impulsive increase of the rate of reconnection on a time scale of just a few seconds in the solar case (tens of seconds in the magnetotail) and may consist of several bursts, associated with pulsating reconnection and multiple reconnection sites. As illustrated by Fig. 8, it is easy to understand that a downward velocity pulse away from the apex of a closed field line causes primarily a compressional pulse in the low guide field, low shear, high- $\beta$, case, whereas for strong guide field this pulse is more Alfvénic, associated with a Poynting flux. If we adopt the transport model suggested by Fletcher and Hudson (2008) we find another interesting analogy between this transport and so-called bursty bulk flows (BBFs) in the magnetotail (Baumjohann et al., 1990; Angelopoulos et al., 1992), which have been shown to provide the major transport of magnetic flux and energy in the magnetotail (Angelopoulos et al., 1994), caused by magnetic reconnection.

An important part of the energy release and conversion process is the division of the released energy into thermal and nonthermal particle energy. In the solar flare scenario this division is usually made on the basis of inferred electron distribution functions, which can be fitted by a ("thermal") Maxwellian and a ("nonthermal") power law tail. Based on hard X-ray (HXR) and $\gamma$-ray luminosities, the total energy of the accelerated (nonthermal) electron and ion populations with energies above $\sim 25 \mathrm{keV}$ is estimated to be as high as $50 \%$ of the total energy released (Miller et al., 1997; Emslie et al., 2005). In the magnetotail substorm scenario such a clear distinction between thermal and nonthermal populations is usually not possible. However, it has also been demonstrated that the increase of energetic particle fluxes above, say, 10-40 keV, observed in the inner tail, can contribute significantly to the total pressure increase (Birn et al., 1997a).

Whether or not such energization can be understood, at least in principle, on the basis of MHD simulations depends critically on the underlying acceleration process. In the magnetotail substorm scenario this process has been demonstrated to be associated with betatron (Birn et al., 1997b, 1998; Li et al., 1998; Zaharia et al., 2000) and Fermi acceleration (Birn et al., 2004) from the collapse of the closed magnetic field lines as a consequence of reconnection, rather than with acceleration at the reconnection site itself. In the solar flare context the acceleration mechanism is not yet understood. In a common picture, the energization takes place in the corona, by a yet unidentified process, whereupon an electron beam is formed that hits the chromosphere and generates hard X-rays by bremsstrahlung. However it is not clear how the large number of accelerated particles inferred from this model can be produced over very short time scales and from a relatively modest reservoir of coronal particles.

If the energization results from direct acceleration by parallel electric fields along an $\mathrm{x}$-line or separator, this process, which can be understood as Joule dissipation, is not adequately described by the resistive MHD model. However, our PIC simulations showed that this energization is only a minor part of the conversion process, even in the extremely localized region covered by the particle simulation. While this finding was made for a mass ratio $m_{i} / m_{e}=25$, we expect that the role of dissipation becomes even further reduced when the electron mass is lowered and the size of the dissipation region decreases. (An investigation of this effect is underway.) We therefore conclude that direct acceleration contributes only insignificantly to the overall energization. A similar conclusion can be made for the conversion of magnetic to bulk kinetic energy, which was found insignificant in both MHD and PIC simulations. The dominant energy output 
from reconnection therefore is expected to be in the form of either Poynting flux, which requires a subsequent conversion to particle energy, as in the model of Fletcher and Hudson (2008), or in the form of the enthalpy flux. Both of these output fluxes are contained in the MHD model although the effects on the particle distribution functions cannot be inferred from this model.

The conversion of the energy fluxes to particle energy may involve further compression, for instance, within collapsing magnetic loops (Somov and Kosugi, 1997; Karlicky and Kosugi, 2004; Giuliani et al., 2005), similar to the mechanism in the magnetotail. Alternatively, dissipation of electromagnetic energy flux might involve nonadiabatic processes in the chromosphere at the bottom of flare loops (Fletcher and Hudson, 2008). The acceleration within collapsing magnetic flux tubes, which undoubtedly takes place, provides another analogy between substorms and flares.

Acknowledgements. This work was performed under the auspices of the US Department of Energy, supported by NASA's Heliophysics Theory and Supporting Research and Technology programs. The authors thank L. Fletcher and T. Neukirch for valuable discussions.

Topical Editor R. Nakamura thanks two anonymous referees for their help in evaluating this paper.

\section{References}

Angelopoulos, V., Baumjohann, W., Kennel, C. F., Coroniti, F. V., Kivelson, M. G., Pellat, R., Walker, R. J., Lühr, H., and Paschmann, G.: Bursty bulk flows in the inner central plasma sheet, J. Geophys. Res., 97, 4027-4039, 1992.

Angelopoulos, V., Kennel, C. F., Coroniti, F. V., Pellat, R., Kivelson, M. G., Walker, R. J., Russell, C. T., Baumjohann, W., Feldman, W. C., and Gosling, J. T.: Statistical characteristics of bursty bulk flow events, J. Geophys. Res., 99, 21257-21280, 1994.

Baker, D. N., Pulkkinen, T. I., Hesse, M., and McPherron, R. L.: A quantitative assessment of energy storage and release in the Earth's magnetotail, J. Geophys. Res., 102, 7159-7168, 1997.

Baumjohann, W. and Nakamura, R.: Observations of tail reconnection, in: Reconnection of Magnetic Fields: MHD and Collisionless Theory and Observations, edited by: Birn, J. and Priest, E. R., p. 209, Cambridge University Press, Cambridge, England, 2007.

Baumjohann, W., Paschmann, G., and Lühr, H.: Characteristics of high-speed ion flows in the plasma sheet, J. Geophys. Res., 95, 3801-3809, 1990.

Birn, J. and Hesse, M.: Energy release and conversion by reconnection in the magnetotail, Ann. Geophys., 23, 3365-3373, 2005, http://www.ann-geophys.net/23/3365/2005/.

Birn, J. and Schindler, K.: Thin current sheets in the magnetotail and the loss of equilibrium, J. Geophys. Res., 107, SMP18, doi:10.1029/2001JA0291, 2002.

Birn, J., Goldstein, H., and Schindler, K.: A theory of the onset of solar eruptive processes, Solar Phys., 57, 81-101, 1978.
Birn, J., Iinoya, F., Brackbill, J. U., and Hesse, M.: A comparison of MHD simulations of magnetotail dynamics, Geophys. Res. Lett., 23, 323-325, 1996.

Birn, J., Thomsen, M. F., Borovsky, J. E., Reeves, G. D., McComas, D. J., and Belian, R. D.: Characteristic plasma properties during dispersionless substorm injections at geosynchronous orbit, J. Geophys. Res., 102, 2309-2324, 1997a.

Birn, J., Thomsen, M. F., Borovsky, J. E., Reeves, G. D., McComas, D. J., Belian, R. D., and Hesse, M.: Substorm ion injections: Geosynchronous observations and test particle orbits in threedimensional dynamic MHD fields, J. Geophys. Res., 102, 23252341, $1997 b$.

Birn, J., Thomsen, M. F., Borovsky, J. E., Reeves, G. D., McComas, D. J., Belian, R. D., and Hesse, M.: Substorm electron injections: Geosynchronous observations and test particle simulations, J. Geophys. Res., 103, 9235-9248, 1998.

Birn, J., Forbes, T. G., and Schindler, K.: Models of three-dimensional flux ropes, Astrophys. J., 588, 578-585, doi:10.1086/373921, 2003.

Birn, J., Thomsen, M. F., and Hesse, M.: Electron acceleration in the dynamic magnetotail: Test particle orbits in threedimensional magnetohydrodynamic simulation fields, Phys. Plasmas, 11, 1825, doi:10.1063/1.1704641, 2004.

Birn, J., Galsgaard, K., Hesse, M., Hoshino, M., Huba, J., Lapenta, G., Pritchett, P. L., Schindler, K., Yin, L., Büchner, J., Neukirch, T., and Priest, E. R.: Forced magnetic reconnection, Geophys. Res. Lett., 32, L06105, doi:10.1029/2004GL022058, 2005.

Birn, J., Forbes, T. G., and Hesse, M.: Stability and dynamical evolution of three-dimensional flux ropes, Astrophys. J., 645, 732741, doi:10.1086/504280, 2006.

Birn, J., Fletcher, L., Hesse, M., and Neukirch, T.: Energy release and transfer in solar flares: Simulations of three-dimensional reconnection, Astrophys. J., in press, 2009.

Carmichael, H.: A Process for Flares, in: AAS/NASA Symposium on the Physics of Solar Flares, edited by: Hess, W. N., p. 451, NASA, Washington, D.C., 1964.

Drake, J. F. and Shay, M. A.: Fundamentals of collisionless reconnection, in: Reconnection of Magnetic Fields: MHD and Collisionless Theory and Observations, edited by: Birn, J. and Priest, E. R., p. 87, Cambridge University Press, Cambridge, England, 2007.

Emslie, A. G., Dennis, B. R., Holman, G. D., and Hudson, H. S.: Refinements to flare energy estimates: A followup to "Energy partition in two solar flare/CME events" by A. G. Emslie et al., J. Geophys. Res. (Space Phys.), 110, 11103, doi: 10.1029/2005JA011305, 2005.

Fletcher, L. and Hudson, H. S.: Impulsive Phase Flare Energy Transport by Large-Scale Alfvén Waves and the Electron Acceleration Problem, Astrophys. J., 675, 1645-1655, doi:10.1086/ 527044, 2008.

Forbes, T. G.: Numerical simulation of a catastrophe model for coronal mass ejections, J. Geophys. Res., 95, 11919-11931, 1990.

Forbes, T. G.: Arcade flare models, in: Magnetohydrodynamic Phenomena in the Solar Atmosophere - Prototypes of Stellar Magnetic Activity, edited by: Uchida, Y., Kosugi, T., and Hudson, H. S., p. 287, Kluwer, 1996.

Gary, G. A.: Plasma beta above solar active regions: rethinking the paradigm, Solar Phys., 203, 71-86, 2001. 
Giuliani, P., Neukirch, T., and Wood, P.: Particle motion in collapsing magnetic traps in solar flares. I. Kinematical theory of collapsing magnetic traps, Astrophys. J., 635, 636-646, 2005.

Hesse, M.: Diffusion region physics, in: Reconnection of Magnetic Fields: MHD and Collisionless Theory and Observations, edited by: Birn, J. and Priest, E. R., p. 108, Cambridge University Press, Cambridge, England, 2007.

Hesse, M. and Birn, J.: Three-dimensional magnetotail equilibria by numerical relaxation techniques, J. Geophys Res., 98, 39733982, 1993.

Hesse, M., Schindler, K., Birn, J., and Kuznetsova, M.: The Diffusion Region in Collisionless Magnetic Reconnection, Phys. Plasmas, 6, 1781-1795, doi:10.1063/1.873436, 1999.

Hesse, M., Birn, J., and Kuznetsova, M.: Collisionless magnetic reconnection: Electron processes and transport modeling, J. Geophys. Res., 106, 3721-3735, 2001.

Hirayama, T.: Theoretical Model of Flares and Prominences. I: Evaporating Flare Model, Solar Phys., 34, 323-338, 1974.

Karlicky, M. and Kosugi, T.: Acceleration and heating processes in a collapsing magnetic trap, Astron. Astrophys., 419, 1159-1168, 2004.

Kopp, R. A. and Pneuman, G. W.: Magnetic reconnection in the corona and the loop prominence phenomenon, Sol. Phys., 50, 85-98, 1976.

Kuperus, M.: Solar flares and magnetospheric substorms, Solar Phys., 47, 361-363, 1976.
Li, X., Baker, D. N., Temerin, M., and Reeves, G. D.: Simulation of dispersionless injections and drift echoes of energetic electrons associated with substorms, Geophys. Res. Lett., 25, 3763-3766, 1998.

Low, B. C.: Solar activity and the corona, Solar Phys., 167, 217265, 1996.

Miller, J. A., Cargill, P. J., Emslie, A. G., Holman, G. D., Dennis, B. R., LaRosa, T. N., Winglee, R. M., Benka, S. G., and Tsuneta, S.: Critical issues for understanding particle acceleration in impulsive solar flares, J. Geophys. Res., 102, 14631-14660, doi: 10.1029/97JA00976, 1997.

Pritchett, P. L.: Onset of magnetic reconnection, in: Reconnection of Magnetic Fields: MHD and Collisionless Theory and Observations, edited by: Birn, J. and Priest, E. R., p. 121, Cambridge University Press, Cambridge, England, 2007.

Somov, B. V. and Kosugi, T.: Collisionless reconnection and highenergy particle acceleration in solar flares, Astrophys. J., 485, 859-868, 1997.

Sturrock, P. A.: Model of the High-Energy Phase of Solar Flares, Nature, 211, 695-697, 1966.

Vasyliūnas, V. M.: Theoretical models of magnetic field line merging, Rev. Geophys. Space Phys., 13, 303-336, 1975.

Zaharia, S., Cheng, C. Z., and Johnson, J. R.: Particle transport and energization associated with substorms, J. Geophys. Res., 105, 18741-18752, 2000. 